\title{
Critical role of aquaporin-3 in epidermal growth factor-induced migration of colorectal carcinoma cells and its clinical significance
}

\author{
ANG LI ${ }^{1}$, DEHONG LU ${ }^{2}$, YUPENG ZHANG ${ }^{1}$, JIA LI $^{1}$, YU FANG $^{1}$, FEI LI $^{1}$ and JIABANG SUN ${ }^{1}$ \\ Departments of ${ }^{1}$ General Surgery and ${ }^{2}$ Pathology, Xuanwu Hospital, Capital Medical University, Beijing 100053, P.R. China
}

Received August 4, 2012; Accepted October 12, 2012

DOI: $10.3892 /$ or.2012.2144

\begin{abstract}
Aquaporins (AQPs) are a family of small, integral membrane proteins that have been shown to play an important role in tumor development and metastasis. Several studies have demonstrated that expression of AQP3 contributes to the enhanced migration of epithelial cells and is related to differentiation, metastasis and vascular invasion in lung and gastric cancer. Therefore, we investigated whether AQP3 could enhance human colorectal carcinoma cell migration and we examined the role of AQP3 in the prognosis of colorectal carcinoma. Our results showed that human epidermal growth factor (hEGF) increased the expression of AQP3 and, subsequently, the migration ability of human colorectal carcinoma cells HCT116 in a dose- and time-dependent manner. The enhanced migration ability of HCT116 cells was blocked by the AQP3 inhibitor, $\mathrm{CuSO}_{4}$. Overexpression of AQP3 induced by hEGF was inhibited by a PI3K/AKT inhibitor, LY294002, but the ERK inhibitor U0126 had a minor effect on the hEGFinduced AQP3 upregulation. Immunohistochemical staining of the cancer tissues and corresponding normal tissues showed that AQP3 expression in cancer tissue was higher compared to that in normal tissue. The expression intensity of AQP3 was associated with the differentiation, lymph node and distant metastasis of colorectal carcinoma patients. Our results suggest that AQP3 overexpression could facilitate colorectal carcinoma cell migration and AQP3 may be considered a potential indicator and therapeutic target for colon tumor metastasis and prognosis.
\end{abstract}

\section{Introduction}

Colorectal cancer is the fourth most common cancer in the world $(1,2)$. In spite of radical surgery and chemotherapy, the overall 5-year survival rate remains poor (3). Cancer recur-

Correspondence to: Professor Jiabang Sun, Department of General Surgery, Xuanwu Hospital, Capital Medical University, 45 Chang Chun Street, Beijing 100053, P.R. China

E-mail: jiabangsun@163.com

Key words: aquaporin 3, colorectal carcinoma, migration, epidermal growth factor rence and metastasis after surgery have been recognized as the main causes of the poor outcome of colorectal cancer patients. It has been documented that more than $50 \%$ of patients with colorectal cancer will develop liver metastases (2). Therefore, properly understanding the mechanisms underlying colorectal cancer metastasis as well as identifying new molecular targets for therapeutic agents is critical for the prevention of colorectal cancer metastasis.

Aquaporins (AQPs) are a family of small, integral membrane proteins involved in the selective transport of water across cell membranes (4,5). Accumulating evidence suggests that the expression of AQP3 is tightly correlated with cell proliferation and migration. Hara-Chikuma and Verkman (6) showed the knockout of AQP3 could affect the proliferation and migration ability of keratinocyte and slow the wound healing rate of mouse skin. However, when the expression of AQP3 was upregulated in human keratinocytes by transfection with human AQP3 DNA plasmid, the cell proliferation was increased (7). In human gastric adenocarcinoma cells, AQP3 knockdown inhibited hEGF-induced AQP3 expression and, thus, cell migration and proliferation (8). Inhibition of AQP3 in human esophageal and oral squamous cell carcinoma also suppressed cancer cell proliferation (9). Since the migration of tumor cells is an important step in tumor invasion and metastasis (10), the above findings strongly suggest that inhibition of AQP3 might be useful in the treatment of cancer.

Some studies have shown that AQP1, AQP3 and AQP8 are also expressed in the normal colon (11-13); however, in colorectal carcinoma, the expression of AQP1 and AQP3 is much higher than in normal tissue $(11,14)$, indicating AQPs may be involved in the development of colorectal carcinoma. However, there are few studies concerning the role of AQP3 in colorectal carcinoma thus far. Based on the above findings, we hypothesized that AQP3 is involved in the migration of colorectal carcinoma. Therefore, in the present study, we investigated the relationship between AQP3 and the migration of colorectal carcinoma cells in vitro and analyzed the expression of AQP3 and the differentiation, lymph node and distant metastasis of human colorectal carcinoma.

\section{Materials and methods}

Cell culture. The human colorectal carcinoma cell line HCT116 was purchased from the Shanghai Institutes for Biological Sciences, Chinese Academy of Sciences, and was 
A
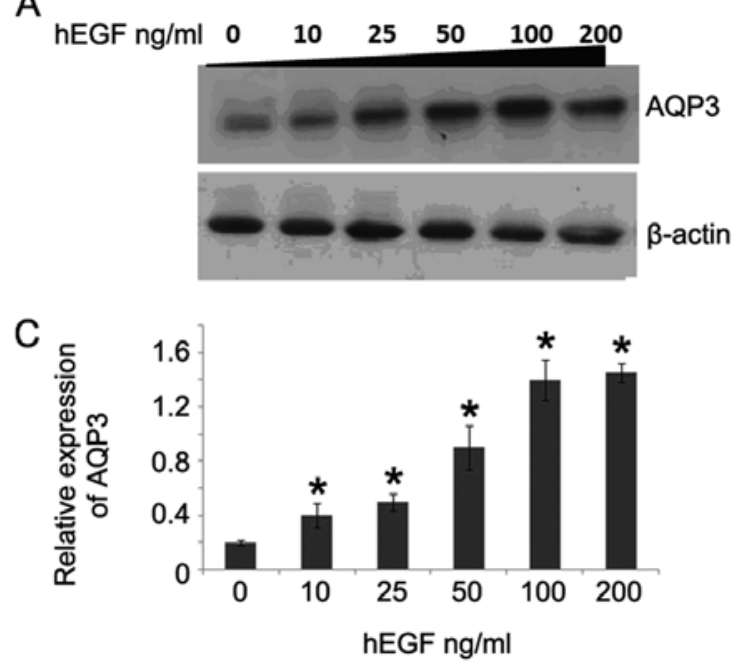

B
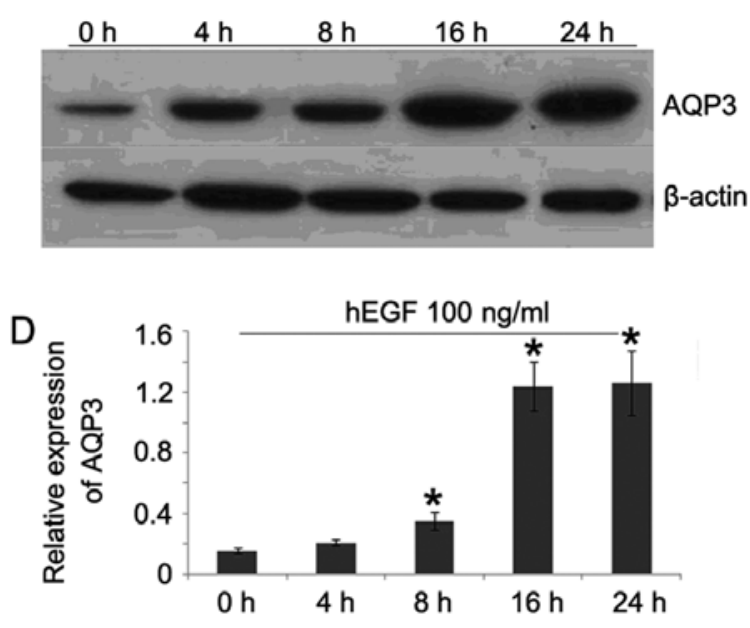

Figure 1. hEGF upregulates AQP3 expression in HCT116 human colorectal carcinoma cells. HCT116 cells were seeded in 6-well plates. After 24 h, the cells were treated with (A) various concentrations of hEGF for another $24 \mathrm{~h}$ or (B) hEGF (100 ng/ml) for different times. Then the protein was extracted and subjected to western blot analysis. (C and D) The quantitative expression of AQP3 in (A and B), respectively. ${ }^{*}<<0.05$, compared with untreated cells.

cultured in RPMI-1640 medium supplemented with 10\% fetal bovine serum (FBS), $100 \mathrm{U} / \mathrm{ml}$ of penicillin and $100 \mathrm{U} / \mathrm{ml}$ of streptomycin, at $37^{\circ} \mathrm{C}, 5 \% \mathrm{CO}_{2}$ and $95 \%$ humidity.

Wound healing assay. Six-well plates were coated with polylysine (PL). HCT116 cells were seeded in the coated plates at a density of $5 \times 10^{5} /$ well and cultured to $\sim 80 \%$ confluence. Wound healing assay was performed as previously described (15). Briefly, a scratch wound was generated by scratching with a $200 \mu$ pipette tip across the center of the well. After scratching, the well was gently washed twice with medium to remove the detached cells and fresh medium was added into the wells. Cells were cultured at $37^{\circ} \mathrm{C}$ for indicated times and images were captured at different time points.

Western blot analysis. Total protein was extracted as previously described (16). Briefly, the cells were lysed with RIPA lysis buffer (Beyotime, Haimen, Jiangsu, China) and then centrifuged at $12,000 \mathrm{xg}$ for $15 \mathrm{~min}$ at $4^{\circ} \mathrm{C}$. The supernatants were collected for the western blot analysis. Protein concentration was determined with the BCA method. Proteins were separated by sodium dodecyl sulfate-polyacrylamide gel electrophoresis and transferred to the polyvinylidene fluoride (PVDF) membrane (Millipore, Billerica, MA, USA). The blot was then probed with primary antibody followed by reaction with horseradish peroxidase-conjugated secondary antibody. The signal was detected using enhanced chemiluminescence and recorded on X-ray film. The relative density of the target bands was quantified using Gel-Pro analyzer version 3.0 (Media Cybernetics, Bethesda, MD, USA).

Patients. Between January 2006 and December 2007, 163 patients (94 men and 69 women; range, 37-80 years, average $66.1 \pm 11.3$ years) with colorectal cancer were enrolled from Xuanwu Hospital of Capital Medical University. Colorectal cancer was diagnosed by colonoscopy and confirmed as adenocarcinoma by pathological examination. There were 4 cases of grade I, 79 cases of grade II, 59 cases of grade III and 21 cases of grade IV according to the American Joint Committee on Cancer (AJCC) staging guidelines (7th edition). All patients received colorectal cancer surgery according to schedule and were followed up for 3 years by telephone or as outpatients. The study was approved by the ethics committee of the Xuanwu Hospital of Capital Medical University.

Immunohistochemistry. The expression of AQP3 was examined by immunohistochemical (IHC) staining. IHC was carried out using a specific mouse polyclonal anti-AQP3 antibody (Bioworld Technology, Minneapolis, MN, USA). The results were scored using the Fromowitz method (17) by two independent pathologists. Briefly, the images were first scored according to the percentage of AQP3 positive cells in the total tumor cells: $\leq 5 \%$, score $0 ; 6-25 \%$, score $1 ; 26-50 \%$, score 2 ; $51-75 \%$, score $3 ;>75 \%$, score 4 . The images were then scored according to the staining depth: negative staining, score 0 ; faint yellow, score 1; brown madder, score 2; dark brown, score 3 . The scores of the same slide were summed to produce a final score: $0-1$ was considered negative (-); 2-3, weakly positive $(+)$; 4-5, moderately positive (++); 6-7, strong positive (+++). Negative and weakly positive were considered as low expression. Moderately positive and strong positive were considered as high expression.

Statistical analysis. Data are expressed as the means \pm SD. Statistical significance was determined using PASW 18.0 for Windows. Student's t-test was used to compare means for two groups and one-way ANOVA was performed for multiple comparisons followed by Newman-Keuls test for multiple comparisons. Strength of IHC was analyzed by Pearson's Chi-squared test. $\mathrm{P}<0.05$ indicated statistically significant differences.

\section{Results}

hEGF upregulates AQP3 expression in HCT116 cells. To study whether hEGF could stimulate AQP3 expression in 
A
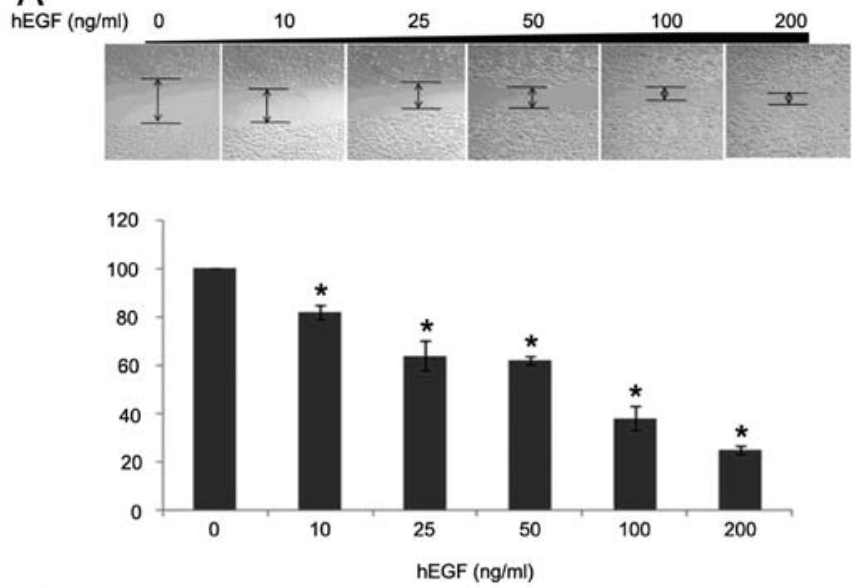

B
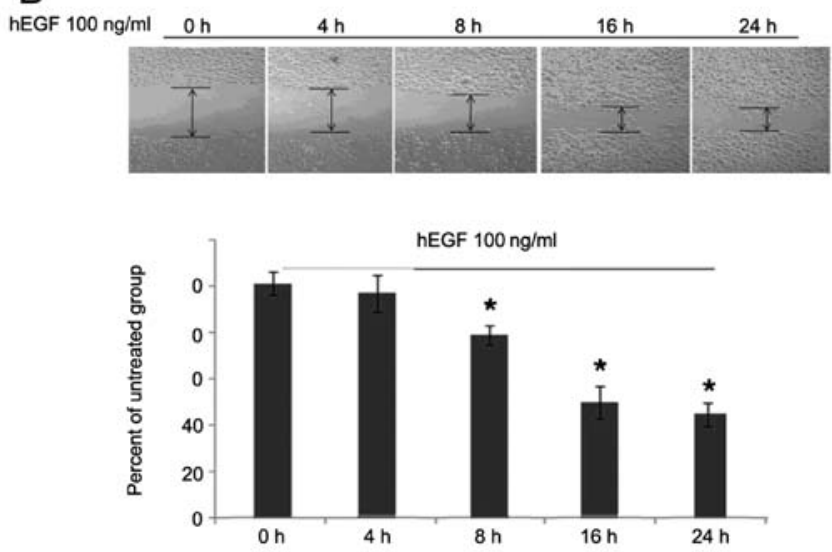

Figure 2. hEGF enhances HCT116 cell migration ability. HCT116 cells were seeded in 6 -well plates at a density of $5 \times 10^{5} /$ well. After $24 \mathrm{~h}$, scratch wounds were generated as described in Materials and methods. Then the cells were treated with (A) various concentrations of hEGF for another $24 \mathrm{~h}$ or (B) hEGF (100 ng/ml) for different times. Images were captured and the wound gaps were determined. The initial length of the wound gap was calculated as $100 \%$. Percent of wound contraction was calculated taking the initial size of the wound gap as $100 \%$. ${ }^{*} \mathrm{P}<0.05$, compared with untreated cells.

human colorectal cancer cells, western blot analysis was performed. As shown in Fig. 1A, after hEGF treatment for $24 \mathrm{~h}$, the expression of AQP3 was significantly increased. The AQP3 expression was increased along with the dose of hEGF, indicating hEGF may upregulate AQP3 expression in a dosedependent manner. Then, we further investigated the AQP3 expression in HCT116 cells after hEGF treatment for different times. The results showed that AQP3 expression was also elevated along with the extension of stimulating time (Fig. 1B and D). These results suggest hEGF was able to upregulate AQP3 expression in a dose- and time-dependent manner.

Upregulation of AQP3 enhances the migration ability of HCT116 cells. Since many studies have shown that overexpression of AQP3 is able to promote cell migration, we considered whether AQP3 could also facilitate the migration of colorectal cancer cells. Following treatment with hEGF, the wound gaps decreased significantly in a time- and dose-dependent manner (Fig. 2). To further elucidate whether the enhanced migra-
A
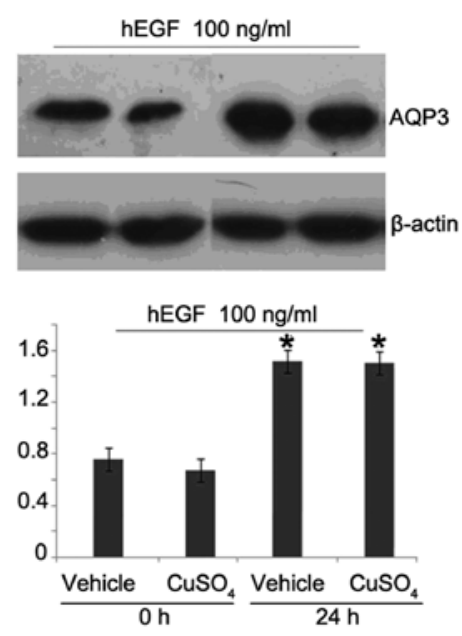

B
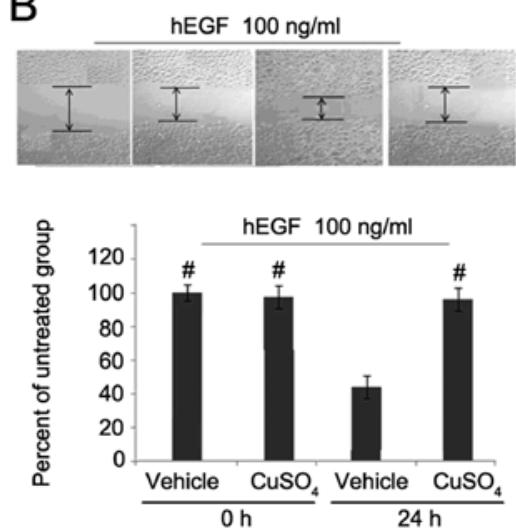

Figure 3. hEGF-induced HCT116 cell migration is inhibited by $\mathrm{CuSO}_{4}$. (A) Expression of AQP3 was not decreased by $\mathrm{CuSO}_{4}$. $\mathrm{HCT} 116$ cells were pretreated with $\mathrm{CuSO}_{4}(500 \mu \mathrm{mol} / \mathrm{l})$ or vehicle for $2 \mathrm{~h}$, and then cultured with or without hEGF $(100 \mathrm{ng} / \mathrm{ml})$ for another $24 \mathrm{~h}$. (B) The migration ability of HCT116 cells was inhibited by $\mathrm{CuSO}_{4}$. HCT116 cells were pretreated with $\mathrm{CuSO}_{4}(500 \mu \mathrm{mol} / \mathrm{l})$ or vehicle for $2 \mathrm{~h}$, and then scratch wounds were generated. The cells were cultured with or without hEGF $(100 \mathrm{ng} / \mathrm{ml})$ for another 24 h. ${ }^{*} \mathrm{P}<0.05$, compared with control cells $(0 \mathrm{~h})$; ${ }^{\#} \mathrm{P}<0.05$, compared with vehicle and hEGF-treated for $24 \mathrm{~h}$.

tion ability of HCT116 cells was due to the upregulation of AQP3 levels, we used a specific inhibitor of AQP3, $\mathrm{CuSO}_{4}$, in the following experiment. After hEGF treatment for $24 \mathrm{~h}$, the expression of AQP3 was not altered by $\mathrm{CuSO}_{4}$ (Fig. 3A). However, the migration ability of HCT116 cells was obviously decreased (Fig. 3B), suggesting that AQP3 plays an important role in the migration of human colorectal cancer cells.

hEGF upregulates AQP3 expression through the PI3K/AKT pathway. Additional experiments were conducted to further investigate the cell signaling pathways involved in hEGFinduced AQP3 expression in human colorectal cancer cells (Fig. 4). Western blot analysis showed that pretreatment of the PI3K/AKT inhibitor LY294002 $(20 \mu \mathrm{M})$ for $1.5 \mathrm{~h}$ almost completely abolished hEGF (100 ng/ml)-induced AQP3 expression. However, pretreatment with the ERK inhibitor, U0126, showed only a slight effect on hEGF-induced AQP3 expression. LY294002 and U0126 did not affect the EGFR expression and phosphorylation. 


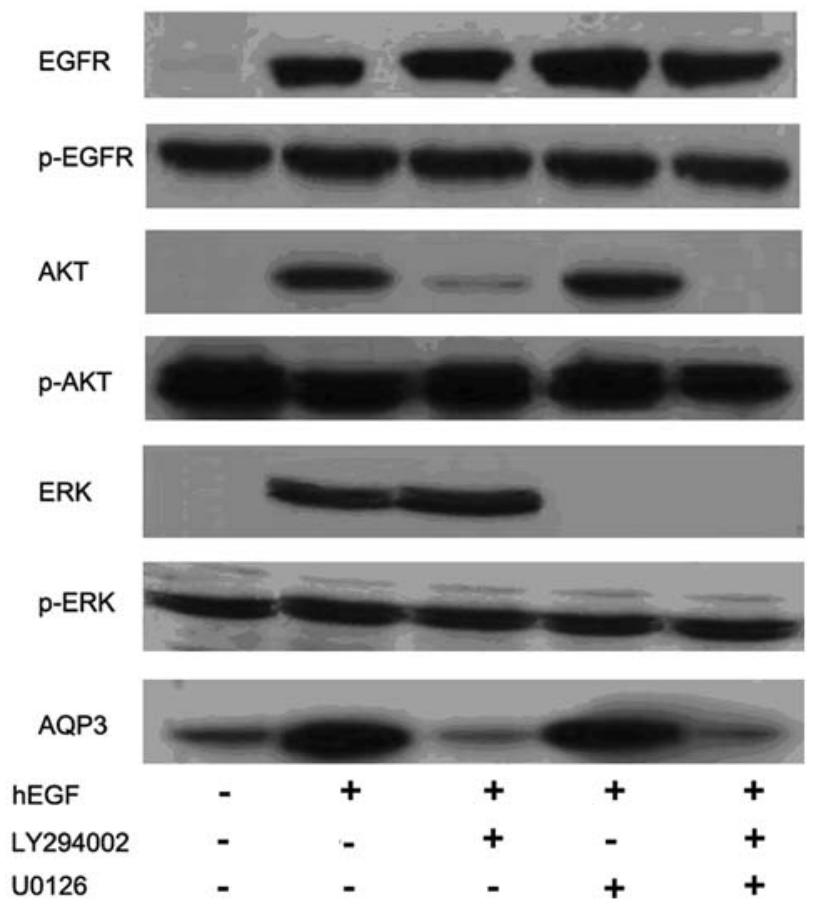

Figure 4. hEGF-induced AQP3 upregulation in HCT116 human colorecta carcinoma cells was abolished by the PI3K/AKT inhibitor. HCT116 cells were seeded in 6-well plates. After $24 \mathrm{~h}$, the cells were pretreated with U0126 $(20 \mu \mathrm{M})$ or LY294002 $(20 \mu \mathrm{M})$ for $1.5 \mathrm{~h}$, and then treated with hEGF $(100 \mathrm{ng} / \mathrm{ml})$ for $24 \mathrm{~h}$. Then the protein was extracted and subjected to western blot analysis.

Expression of AQP3 in colorectal carcinoma tissues and corresponding normal tissues. AQP3 expression was found in both normal colonic epithelium and colorectal carcinoma tissue as shown by IHC staining (Fig. 5). AQP3 protein was found in the membranes and cytoplasm of colonic epithelial cells. In the normal colonic tissues, 119 cases (73.0\%) showed low expression and 44 cases $(27.0 \%)$ showed high expression (Table I); whereas in colorectal carcinoma tissues, 70 cases (42.9\%) showed low expression and 93 cases $(57.1 \%)$ showed high expression. The difference was significant between the carcinoma tissues and normal tissues.

AQP3 expression is associated with differentiation, lymph node and distant metastasis in colorectal carcinoma. Since we found the AQP3 was highly expressed in colorectal carcinoma tissues, we sought to investigate whether there was any clinical value in the AQP3 expression in colorectal carcinoma. Patients were grouped according to the clinical parameters, as shown in Table II. We found that in the high/medium differentiation group, 50.7\% (69/136) of patients showed high expression of AQP3, whereas 77.8\% (21/27) of patients in the low differentiation group showed high expression of AQP3. A significant difference was found between the two groups. Patients with lymph node metastasis showed a higher rate of AQP3 overexpression $(52 / 80,65.0 \%)$ than those without node metastasis $(38 / 83,45.8 \%)$. Similarly, patients with distant metastasis at initial diagnosis $(20 / 21,95.2 \%)$ also exhibited a higher rate of AQP3 overexpression than those without $(70 / 142,49.3 \%)$. However, when the patients were grouped by gender, age and adjacent organ invasion, no significant differences were detected.

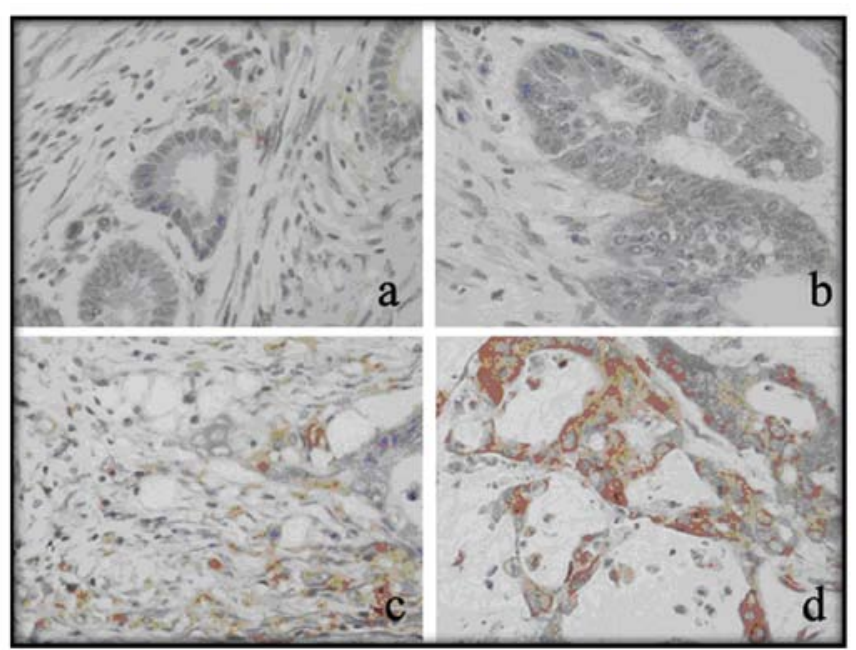

Figure 5. Immunohistochemistry of the expression of AQP3 in colorectal carcinoma tissues and corresponding normal tissues. Representative images of AQP3 expression in colorectal carcinoma tissues and corresponding normal tissues determined by immunohistochemistry. (A) Normal colon, lower expression; (B) moderately differentiated tubular adenocarcinoma, low expression; (C) moderately differentiated tubular adenocarcinoma, high expression; (D) mucinous adenocarcinoma, high expression (magnification, $\mathrm{x} 400$ ).

\section{Discussion}

In the present study, we first showed that hEGF was able to upregulate $\mathrm{AQP} 3$ expression and subsequent migration ability through the PI3K/Akt signaling pathway in human colorectal carcinoma cells. $\mathrm{CuSO}_{4}$, an inhibitor for AQP3 signaling, blocked hEGF-induced upregulation of colorectal carcinoma cell migration, but displayed only a minor effect on AQP3 expression and EGFR expression and activation. We also found the AQP3 expression in colorectal carcinoma tissues was significantly higher than in normal tissues by immunohistochemistry. Significantly, the overexpression of AQP3 was associated with differentiation, lymph node and distant metastasis in patients with colorectal carcinoma.

EGF, which is a single-chain polypeptide separated from male rat submandibular gland in 1962 (18), controls cell proliferation, differentiation, apoptosis and migration through binding to its receptor, EGFR. In normal tissues, there is very low expression of EGF and EGFR, while in many malignant cells, they are usually overexpressed $(19,20)$. Previous studies have found that EGF could induce AQP3 upregulation and cell migration in human ovarian and gastric cancer cells $(8,21)$. Therefore, we assumed that EGF-induced cell migration is also mediated by AQP3. In the present study, we showed that hEGF induced AQP3 upregulation in human colorectal carcinoma cell lines in a time- and dose-dependent manner and increased cell migration. In addition, when the cells were pretreated with $\mathrm{CuSO}_{4}$, the increase of cell migration induced by hEGF was abolished, indicating AQP3 is directly involved in the cell migration.

Ciardiello and Tortora (22) reported that after the activation of EGFR, EGF regulates cell proliferation, adhesion and movement through the downstream PI3K/AKT or MAPK signaling pathways. In our study, the results showed that pretreatment with the ERK inhibitor did not alter the AQP3 expression in 
Table I. Comparison of AQP3 in colorectal carcinoma tissues and corresponding normal tissues.

\begin{tabular}{lccc}
\hline & \multicolumn{2}{c}{ AQP3 } & P-value \\
\cline { 2 - 4 } & Low expression (\%) & High expression (\%) & 0.000 \\
\hline Normal tissues & $119(73.0)$ & $44(27.0)$ & $90(55.2)$ \\
\hline
\end{tabular}

Table II. Correlation between AQP3 expression and multiple clinical parameters.

\begin{tabular}{|c|c|c|c|}
\hline & \multicolumn{2}{|c|}{ AQP3 } & \multirow[b]{2}{*}{ P-value } \\
\hline & Low expression $(\%)$ & High expression (\%) & \\
\hline \multicolumn{4}{|l|}{ Gender } \\
\hline Male & $45(46.9)$ & $51(53.1)$ & 0.631 \\
\hline Female & $28(41.8)$ & $39(58.2)$ & \\
\hline \multicolumn{4}{|l|}{ Age (years) } \\
\hline$<60$ & 18 (42.9) & $24(57.1)$ & 0.366 \\
\hline $60-69$ & $26(53.1)$ & 23 (46.9) & \\
\hline$\geq 70$ & $29(40.3)$ & $43(59.7)$ & \\
\hline \multicolumn{4}{|l|}{ Differentiation } \\
\hline Medium/high & $67(49.3)$ & $69(50.7)$ & 0.011 \\
\hline Low & $6(22.2)$ & $21(77.8)$ & \\
\hline \multicolumn{4}{|l|}{ Adjacent organ } \\
\hline No invasion & $68(45.3)$ & $82(54.7)$ & 0.774 \\
\hline Invasion & $5(38.5)$ & $8(61.5)$ & \\
\hline \multicolumn{4}{|c|}{ Lymph node metastasis } \\
\hline No & $45(54.2)$ & $38(45.8)$ & 0.018 \\
\hline Yes & $28(35.0)$ & $52(65.0)$ & \\
\hline \multicolumn{4}{|c|}{$\begin{array}{l}\text { Distant metastasis } \\
\text { (Initial diagnosis) }\end{array}$} \\
\hline No & $72(50.7)$ & $70(49.3)$ & 0.000 \\
\hline Yes & $1(4.8)$ & $20(95.2)$ & \\
\hline
\end{tabular}

colorectal cancer cells, whereas the PI3K/AKT inhibitor abolished hEGF-induced AQP3 expression in colorectal cancer cells, indicating hEGF-induced AQP3 expression in colorectal cancer cells is mediated by the PI3K/AKT, but not the ERK pathway. Some studies showed AQP3 expression induced by hEGF or HGF was regulated via the ERK signaling pathway in human gastric carcinoma cells $(9,23)$. In another study regarding EGF-induced AQP3 upregulation and cell migration in human ovarian cancer cells, it was indicated that the PI3K and ERK pathways were both involved in EGF-induced cell migration and AQP3 expression (21). The inconsistency may be due to the difference in cancer type.

Tumor cell adhesion and migration play an important role in tumor metastasis. Since overexpression of AQP3 could enhance the migration ability of various tumor cells, it also played a role in the clinical progression of tumors. A retrospective analysis of 149 cases of lung cancer specimens showed the level of AQP3 was correlated with the pathological type, differentiation grade and clinical staging (24). Shen et al (25) found that the expression of AQP3 was higher in undifferentiated gastric carcinoma than in well-differentiated tumors, and was associated with lymph node metastasis and lymphatic vessel invasion. Moon et al (14) detected the expression of AQP3 in 16 cases of colorectal carcinoma tissues and corresponding adjacent normal colon tissue and showed high AQP3 expression in colorectal carcinoma. These results suggest the expression of AQP3 in colorectal carcinoma could affect tumor biological behavior and prognosis. In the present study, we found a stronger AQP3 expression in colorectal carcinoma than in normal tissues in accordance with a previous study (14). Additional analysis showed that AQP3 expression was associated with differentiation, lymph node and distant metastasis in colorectal carcinoma. In the patients with a low differentiation grade, lymph node or distant metastasis, high 
level of AQP3 was often observed. These clinical data suggest high expression of AQP3 predicts tumor metastasis and poor prognosis in colorectal carcinoma.

In conclusion, our results suggest AQP3 overexpression in human colorectal carcinoma cells could facilitate cell migration. The PI3K/AKT, but not the ERK, signaling pathway is involved in hEGF-induced AQP3 expression. AQP3 was overexpressed in colorectal carcinoma tissues compared with that in corresponding normal tissues and correlated with the differentiation grade, lymph node and distant metastasis of the tumor. Our results suggest an important role of AQP3 in human colorectal carcinoma as a potential indicator and therapeutic target for colon tumor metastasis and prognosis. Our data also indicate hEGF may associate poor prognosis of colorectal carcinoma and contribute to potential therapeutic strategies for the prevention of colon tumor metastasis.

\section{References}

1. Remontet L, Esteve J, Bouvier AM, et al: Cancer incidence and mortality in France over the period 1978-2000. Rev Epidemiol Sante Publique 51: 3-30, 2003.

2. Jemal A, Siegel R, Ward E, Murray T, Xu J and Thun MJ: Cancer statistics, 2007. CA Cancer J Clin 57: 43-66, 2007.

3. Giuliani F, De Vita F, Colucci G and Pisconti S: Maintenance therapy in colon cancer. Cancer Treat Rev 36 (Suppl 3): S42-S45, 2010.

4. Agre P: The aquaporin water channels. Proc Am Thorac Soc 3: $5-13,2006$.

5. Magni F, Sarto C, Ticozzi D, et al: Proteomic knowledge of human aquaporins. Proteomics 6: 5637-5649, 2006.

6. Hara-Chikuma M and Verkman AS: Aquaporin-3 facilitates epidermal cell migration and proliferation during wound healing. J Mol Med 86: 221-231, 2008.

7. Nakahigashi K, Kabashima K, Ikoma A, Verkman AS, Miyachi Y and Hara-Chikuma M: Upregulation of aquaporin-3 is involved in keratinocyte proliferation and epidermal hyperplasia. J Invest Dermatol 131: 865-873, 2011.

8. Huang Y, Zhu Z, Sun M, et al: Critical role of aquaporin-3 in the human epidermal growth factor-induced migration and proliferation in the human gastric adenocarcinoma cells. Cancer Biol Ther 9: 1000-1007, 2010.

9. Kusayama M, Wada K, Nagata M, et al: Critical role of aquaporin 3 on growth of human esophageal and oral squamous cell carcinoma. Cancer Sci 102: 1128-1136, 2011.
10. Bogenrieder T and Herlyn M: Axis of evil: molecular mechanisms of cancer metastasis. Oncogene 22: 6524-6536, 2003.

11. Fischer H, Stenling R, Rubio C and Lindblom A: Differential expression of aquaporin 8 in human colonic epithelial cells and colorectal tumors. BMC Physiol 1: 1, 2001.

12. Ishibashi K, Sasaki S, Saito F, Ikeuchi T and Marumo F: Structure and chromosomal localization of a human water channel (AQP3) gene. Genomics 27: 352-354, 1995.

13. Hasegawa H, Lian SC, Finkbeiner WE and Verkman AS: Extrarenal tissue distribution of CHIP28 water channels by in situ hybridization and antibody staining. Am J Physiol 266: C893-C903, 1994.

14. Moon C, Soria JC, Jang SJ, et al: Involvement of aquaporins in colorectal carcinogenesis. Oncogene 22: 6699-6703, 2003.

15. Kim M, Yoon S, Lee S, et al: Gremlin-1 induces BMP-independent tumor cell proliferation, migration, and invasion. PLoS One 7: e35100, 2012.

16. Du J, Cheng B, Zhu X and Ling C: Ginsenoside Rg1, a novel glucocorticoid receptor agonist of plant origin, maintains glucocorticoid efficacy with reduced side effects. J Immunol 187: 942-950, 2011.

17. Fromowitz FB, Viola MV, Chao S, et al: ras $\mathrm{p} 21$ expression in the progression of breast cancer. Hum Pathol 18: 1268-1275, 1987.

18. Cohen S and Elliott GA: The stimulation of epidermal keratinization by a protein isolated from the submaxillary gland of the mouse. J Invest Dermatol 40: 1-5, 1963.

19. Pryczynicz A, Guzinska-Ustymowicz K, Kemona A and Czyzewska J: Expression of EGF and EGFR strongly correlates with metastasis of pancreatic ductal carcinoma. Anticancer Res 28: 1399-1404, 2008.

20. Jin Y, Li JP, Tang LY, et al: Protein expression and significance of VEGF, EGFR and MMP-9 in non-small cell lung carcinomas. Asian Pac J Cancer Prev 12: 1473-1476, 2011.

21. Ji C, Cao C, Lu S, et al: Curcumin attenuates EGF-induced AQP3 up-regulation and cell migration in human ovarian cancer cells. Cancer Chemother Pharmacol 62: 857-865, 2008.

22. Ciardiello F and Tortora G: A novel approach in the treatment of cancer: targeting the epidermal growth factor receptor. Clin Cancer Res 7: 2958-2970, 2001.

23. Wang J, Gui Z, Deng L, et al: c-Met upregulates aquaporin 3 expression in human gastric carcinoma cells via the ERK signalling pathway. Cancer Lett 319: 109-117, 2012.

24. Liu YL, Matsuzaki T, Nakazawa T, et al: Expression of aquaporin 3 (AQP3) in normal and neoplastic lung tissues. Hum Pathol 38: 171-178, 2007.

25. Shen L, Zhu Z, Huang Y, et al: Expression profile of multiple aquaporins in human gastric carcinoma and its clinical significance. Biomed Pharmacother 64: 313-318, 2010. 